\title{
Mirror-Image Dextrocardia
}

\author{
Brent M. McGrath MD MSc PHD', Peter West MD FRCPC ${ }^{2}$ \\ ${ }^{1}$ Associate Chief Resident (2012 - 2013), Department of Medicine, Saint John Regional Hospital; \\ Class of 2010, Faculty of Medicine, Dalhousie University \\ ${ }^{2}$ Department of Medicine, General Internal Medicine and Critical Care, Saint John Regional Hospital
}

\begin{abstract}
There are several variants of a rightward-positioned heart. In mirror-image dextrocardia, the anterior-posterior relationship of the cardiac chambers and great vessels are maintained; however, their left-to-right orientation is reversed. This form of dextrocardia most often occurs in the context of situs inversus totalis in which the other major visceral organs (including the stomach and liver) are reversed. Mirror-image dextrocardia is largely regarded as a normal variant with intracardiac malformations and pathology no more frequent than those of the general population. Physical exam, CXR, and ECG are diagnostic of mirror-image dextrocardia.
\end{abstract}

\section{Case Presentation}

A 20-year-old woman is admitted to the internal medicine service for treatment of a post-viral encephalomyelitis. Initial history reveals a previously well young woman with no reported history of cardiovascular disease or current symptoms. The patient has a thin chest wall and heart sounds are heard over the entire precordium, with no extra heart sounds or murmurs appreciated. The characteristics of the patient's apical beat were not reported. While admitted to hospital, the patient underwent a posterior-anterior chest radiograph, which was clearly abnormal (Fig. 1). To further delineate the features on chest radiograph, an electrocardiogram (ECG) is ordered (Fig. 2). The ECG is clearly abnormal. A repeat ECG is done minutes after the first and is normal (Fig. 3). What is the ECG diagnosis?

\section{Discussion}

The ECG shown in Figure 2 is from a typical left-sided ECG recording. Interpretation reveals tachycardia at a rate of approximately 105 beats per minute. The PR (128 msec) and QTc $(436 \mathrm{msec})$ intervals are both within normal limits. A right axis deviation is observed. The differential diagnosis for a right axis deviation is short, including myocardial infarction of the lateral wall, right ventricular hypertrophy, left posterior fascicular block, vertically positioned heart, chronic obstructive pulmonary disease, pulmonary embolus, limb lead reversal and dextrocardia. Further analysis reveals inverted $\mathrm{P}$ and $\mathrm{T}$ waves in Lead I. Lead aVR has a positive QRS, while aVL exhibits a negative

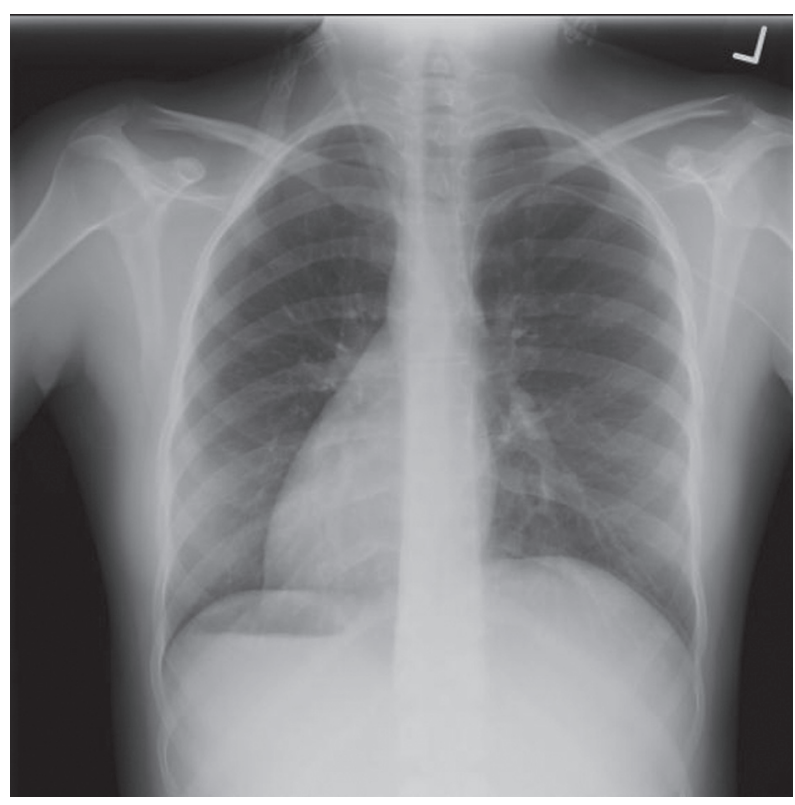

Figure 1. Posterior-anterior chest radiograph.

QRS (suggesting a left posterior fascicular block) and inverted $\mathrm{P}$ and $\mathrm{T}$ waves. The precordial leads are also abnormal with V2 appearing more characteristic of V1 with an inverted $\mathrm{T}$ wave, and vice versa. The progression of the QRS complex is also abnormal and appears reversed, with reduction in amplitude from V1 - V6, particular in the left-sided leads V4 - V6. There is also $\mathrm{T}$ wave inversion throughout the precordial leads. In isolation, the limb lead findings are suggestive of limb lead reversal; however, in the context of the findings in the precordial leads the most likely diagnosis is mirrorimage dextrocardia. 


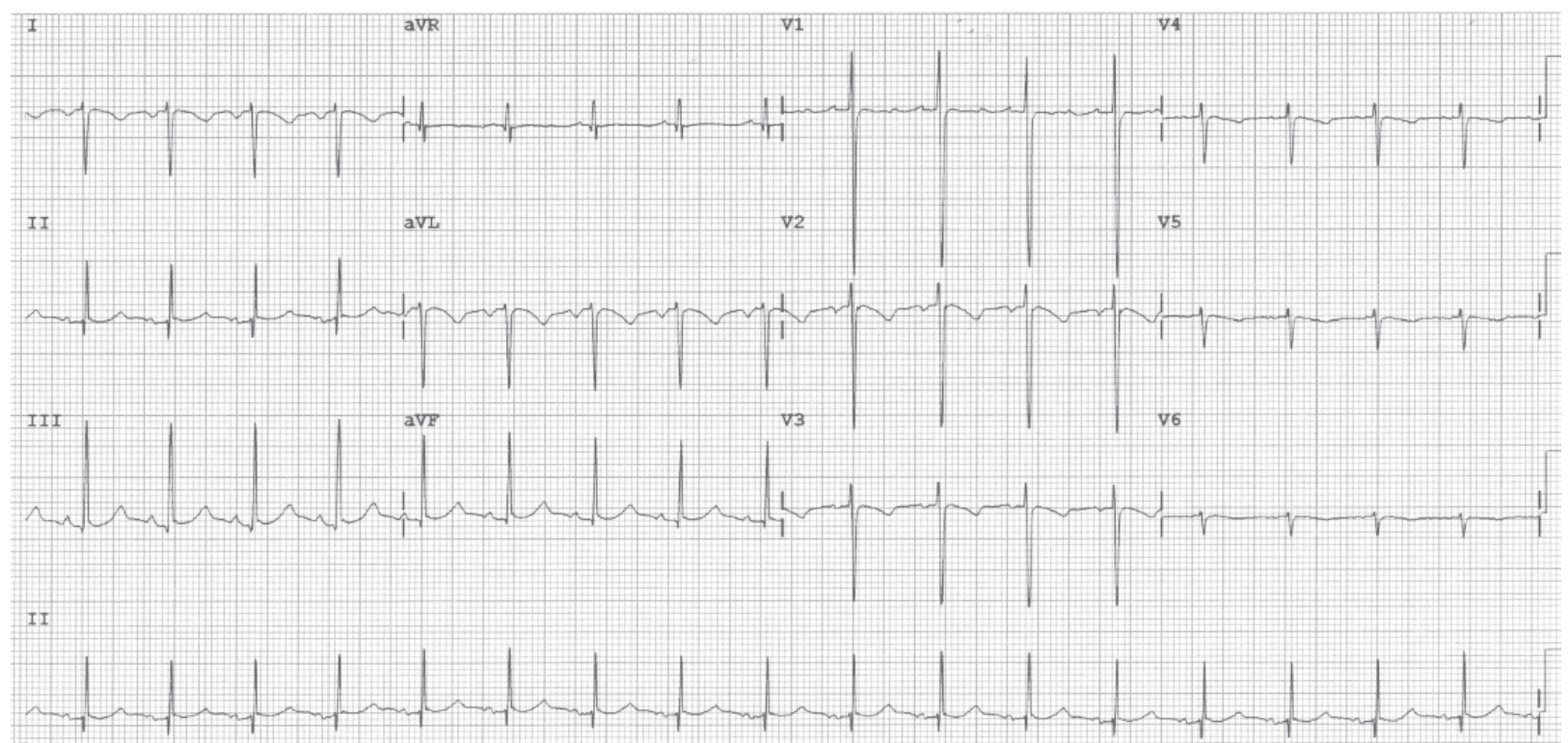

Fig. 2. ECG of a 20-year-old woman.

The posterior-anterior chest radiograph in Figure 1 reveals a right-sided heart (i.e., dextrocardia). Furthermore, note the gastric bubble in the right upper abdominal quadrant and an elevated left hemi-diaphragm findings characteristic of situs inversus totalis.

The ECG depicted in Figure 3 was recorded in the same patient, only moments after that in Figure 2. Given the presence of dextrocardia, a right-sided ECG was done with both the limb and precordial leads reversed. The resultant ECG reveals normal sinus tachycardia with no other abnormalities noted.

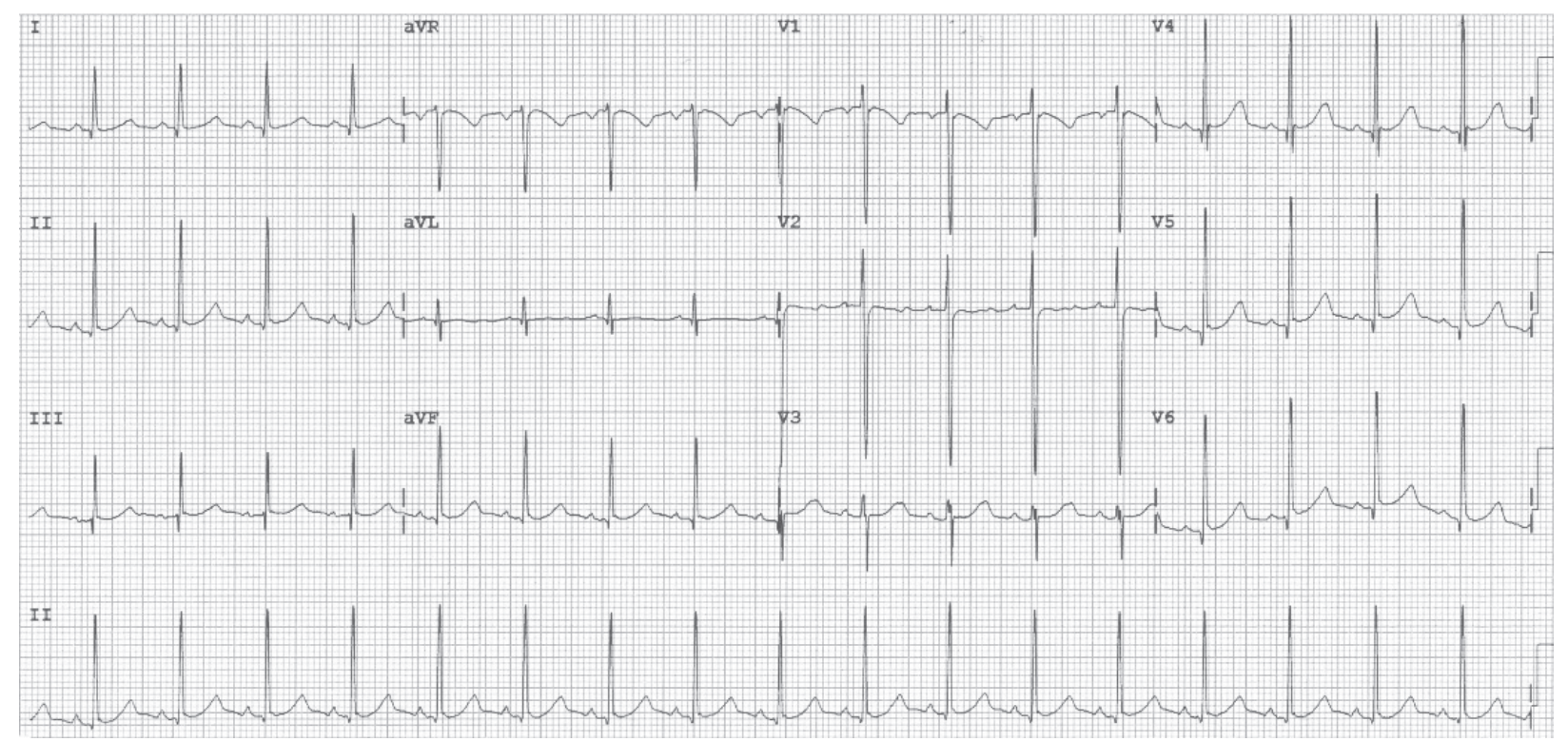

Fig. 3. ECG of the same 20-year-old woman, done minutes after the first (Fig. 2).
In this case, CXR and ECG's are diagnostic of mirrorimage dextrocardia in the context of situs inversus totalis. ${ }^{1,2}$ On ausculation, the patient's heart sounds were largely unhelpful, most likely owing to her thin chest wall. As stated above, while not reported, it is likely that she would have had a right-sided apical impulse. In mirror-image dextrocardia, the anterior-posterior relationship of the cardiac chambers and great vessels are maintained; however, their left-to-right orientation is reversed. ${ }^{3}$ This form of dextrocardia most often occurs in the context of situs inversus totalis, in which the other major visceral organs (including the stomach and liver) are reversed. Mirror-image dextrocardia is 
largely regarded as a normal variant with intracardiac malformations and pathology no more frequent then those of the general population. ${ }^{3}$ The prevalence of this condition is not known. While a normal variant, patients should be informed of their unique anatomy so that they may indicate this anomaly on medical history. This will enable future treating physicians to correlate symptoms and tests with underlying anatomy (i.e., if undergoing cardiac catheterization or when presenting with left upper quadrant pain or left lower quadrant pain in the context of cholecystitis or appendicitis, respectively).

There are two variants of a rightward-positioned heart, which differ depending on the anatomical positioning of the specific heart chambers, great vessels and other visceral organs. Dextroversion refers to a right-sided heart in the context of situs solitus (i.e. only the heart is malpositioned). ${ }^{1,3,4}$ Dextroversion is often associated with underlying clinically important cardiovascular anomalies. Dextroposition refers to a rightward displaced heart (with apex left) due to an extra-cardiac aetiology, most commonly, pulmonary. ${ }^{1,4}$ It is the cause of the dextroposition - not the dextroposition itself - that may or may not have clinical importance. Both can be distinguished based upon their own unique clinical, radiographic and ECG characteristics. A third clinical entity that warrants mention is Kartagener's syndrome. This syndrome is a type of primary ciliary dyskinesia and is characterized by the triad of situs inversus totalis (including mirror-image dextrocardia), chronic sinusitis and bronchiectasis. ${ }^{5}$ These patients often present with chronic upper and lower respiratory tract infections, as well as sterility in males, all owing to ciliary dysfunction.

Mirror-image dextrocardia is an interesting yet clinically silent phenomenon. These patients should have a normal life expectancy with no specific clinical sequellae; however, it would be important to know if this condition was present in any patient undergoing cardiac catheterization or surgery. It should be part of a differential diagnosis in any patient with suggestive physical findings and the above-described constellation of ECG findings. However, in most patients mirrorimage dextrocardia is most likely to be noted incidentally on chest radiography.

\section{References}

1. Anselmi G, Munoz S, Blanco P, Machado I, de la Cruz MV. Systematization and clinical study of dextroversion, mirror-image dextrocardia, and laevoversion. Br Heart J 1972;34:1085-1098.

2. Spigelman $A D$. Images in clinical medicine: situs inversus. $\mathrm{N}$ Engl J Med 1994;330(9):606.

3. Grant RP. The syndrome of dextroversion of the heart. Circulation 1958;18:25-36.

4. Rogel S, Schwartz A, Rakower J. The differentiation of dextroversion from dextroposition of the heart and their relation to pulmonary abnormalities. Chest 1963;44:186-192.

5. Kapur V, Chauhan S, D'Cruz S, Sachdev A. Kartagener's syndrome. Lancet 2009;373:1973. 